\title{
Impact of Deficiency of Intrinsic Coagulation Factors XI and XII on Ex Vivo Thrombus Formation and Clot Lysis
}

\author{
José W. P. Govers-Riemslag ${ }^{1,2}$ Joke Konings ${ }^{1,2,3}$ Judith M. E. M. Cosemans ${ }^{1}$ Johanna P. van Geffen ${ }^{1}$ \\ Bas de Lat $^{3}$ Johan W. M. Heemskerk ${ }^{1}$ Yesim Dargaud ${ }^{4, *}$ Hugo ten Cate ${ }^{1,2, *}$
}

\footnotetext{
${ }^{1}$ Department of Biochemistry, Cardiovascular Research Institute Maastricht (CARIM), Maastricht University Medical Center, Maastricht, The Netherlands

2 Department of Internal Medicine, Cardiovascular Research Institute Maastricht (CARIM), Maastricht University Medical Center,

Maastricht, The Netherlands

${ }^{3}$ Synapse Research Institute, Cardiovascular Research Institute Maastricht (CARIM), Maastricht University, Maastricht, The Netherlands

${ }^{4}$ Unité d 'Hémostase Clinique, Hôpital Cardiologique Louis Pradel, Hospices Civils de Lyon, Bron, France
}

Address for correspondence Joke Konings, PhD, Synapse Research Institute, CARIM, Maastricht University, Maastricht, Oxfordlaan 70, 6229 EV Maastricht, The Netherlands (e-mail: J.Konings@thrombin.com).

TH Open 2019;3:e273-e285.

\begin{abstract}
Keywords

- clot lysis

- factor XII

- factor XI

- deficiency

- thrombus formation

The contributions of coagulation factor XI (FXI) and FXII to human clot formation is not fully known. Patients with deficiency in FXI have a variable mild bleeding risk, whereas FXII deficiency is not associated with bleeding. These phenotypes make FXII and FXI attractive target proteins in anticoagulant therapy. Here, we studied the mechanisms of fibrin clot formation, stability, and fibrinolytic degradation in patients with severe FXI or FXII deficiency. Thrombin generation was triggered in platelet-poor (PPP) and platelet-rich plasma (PRP) with the biological FXII trigger sulfatides. Intrinsic and extrinsic thrombus formation and degradation in whole blood were determined with rotational thromboelastometry (ROTEM). Clot formation under flow was assessed by perfusion of whole blood over collagen microspots with(out) tissue factor (TF). Thrombin generation and clot formation were delayed in FXII- and FXI-deficient patients triggered with sulfatides. In FXI-deficient plasma, this delay was more pronounced in PRP compared to PPP. In whole blood of FXIIdeficient patients, clots were smaller but resistance to fibrinolysis was normal. In whole blood of FXI-deficient patients, clot formation was normal but the time to complete fibrinolysis was prolonged. In flow chamber experiments triggered with collagen/TF, platelet coverage was reduced in severe compared with moderate FXI deficiency, and fibrin formation was impaired. We conclude that quantitative defects in FXII and FXI have a substantial impact on contact activation-triggered coagulation. Furthermore, FXI deficiency has a dose-dependent suppressing effect on flow-mediated and platelet/TF-dependent clot formation. These last data highlight the contribution of particularly FXI to hemostasis.
\end{abstract}

\section{Introduction}

Thrombosis is a critical element in the pathogenesis of ischemic cardiovascular disease as well as in venous thromboem-

* These authors contributed equally to this study.

received

November 27, 2018

accepted after revision

June 7, 2019
DOI https://doi.org/

10.1055/s-0039-1693485. ISSN 2512-9465. bolism. Both epidemiological and animal studies indicate that the intrinsic coagulation factors, factor XI (FXI) and factor XII (FXII), contribute to the development of pathological thrombus formation. ${ }^{1-4}$ In mice, congenital deficiency of either F11 or F12 protected against experimentally induced arterial thrombosis. ${ }^{5-7}$ Since these deficiencies are not associated
License terms

Stuttgart · New York

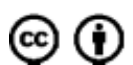


with bleeding (FXII) or with a mild bleeding phenotype (FXI), respectively, the contribution of these intrinsic factors in normal hemostasis is limited. Moreover, antibody-, antisense-, or aptamer-based inhibitors of FXI or FXII showed promising, suppressive effects in several in vivo models of arterial thrombosis. ${ }^{8-13}$ Also, in primate thrombosis models, immunological inhibition of FXI or FXII appeared to be thromboprotective. ${ }^{10}$ Limited and also conflicting information is available on how reduced levels of FXI and FXII can protect against thrombosis in man, since subjects with a congenital deficiency in either factor still experience cardiovascular events. ${ }^{14} \mathrm{~A}$ recent trial indicated that antisense FXI treatment reduced postoperative venous thromboembolism without increasing bleeding in patients undergoing knee replacement surgery. ${ }^{15}$ Although the collective data point to a more prominent role of human FXI and FXII in thrombosis than in hemostasis, it is important to better understand the mechanisms and processes that are regulated by these contact activation factors.

The thrombus formation process involves different pathways that interact with FXI and FXII. Next to a relatively slow triggering via the intrinsic FXII pathway, thrombin generation is potently triggered by the fast, extrinsic tissue factor (TF)/FVIIa pathway. First traces of formed thrombin then feedback to activate platelets and proteolyse coagulation (co)factors, including FXI. The latter process leads to a gradual build-up of sufficiently high thrombin levels to support formation of a fibrin clot. ${ }^{16}$ Activated, phosphatidylserine-exposing platelets enhance these reactions to promote both thrombin generation and fibrin formation. Furthermore, there are indications for cross-talk between the intrinsic and extrinsic coagulation pathways, with FXI as an essential element, but under which conditions these become prominent is unclear. Recent evidence also suggests that FXI can be activated on activated platelets, independently of FXII. ${ }^{17,18}$ Moreover, FXIla can interact with fibrin(ogen) resulting in a tighter clot structure and altered fibrinolysis. $^{19-21}$

The aim of this study, in patients with a congenital deficiency in one of the contact system proteins, is to assess the contribution of FXI and FXII in (patho)physiologically relevant integrative measurements of coagulation activity, i.e., thrombin and fibrin clot generation in plasma and blood under stasis, formation of a fibrin-thrombus under flow conditions, and the degradation of formed clots.

\section{Material and Methods}

\section{Patients and Healthy Subjects}

Included in the study were well-characterized patients with established deficiencies in FXI or FXII and healthy control donors, who were examined in parallel. The study population concerned six patients with FXI deficiency and four patients with FXII deficiency, along with 10 controls subjects of comparable age. The study was designed and performed according to the Helsinki declaration and each subject provided written informed consent. The study was approved by the Lyon University Hospital's medical ethics committee under number ID-RCB 2013-A01274-41.
Overall characteristics of patients and control subjects are indicated in - Table 1. Of the FXI-deficient patients, four had a severe $(\mathrm{FXI} \leq 5 \mathrm{IU} / \mathrm{dL})$, one a moderate (FXI $6-15 \mathrm{IU} / \mathrm{dL}$ ), and one a mild (FXI $>15$ and $<60 \mathrm{IU} / \mathrm{dL}$ ) deficiency. All FXII-deficient patients had a severe deficiency $(\mathrm{FXII} \leq 5 \mathrm{IU} / \mathrm{dL})$. All patients had initially been identified based on a prolonged activated partial thromboplastin time (aPTT). None of the FXIIdeficient patients had experienced any bleeding or thrombosis problems. Two of the six FXI-deficient patients ( 1 and $4 \mathrm{IU} / \mathrm{dL}$ ) had experienced major bleeding, three of the others had a mild bleeding tendency, and one FXI-deficient patient bruises easily. The 10 healthy controls had no personal history of bleeding or thrombosis. Subjects had not received medication affecting platelets or coagulation, including oral contraceptives or anti-inflammatory drugs (NSAIDs), at least during 14 days before blood collection.

\section{Blood Collection and Processing}

Blood was collected into S-Monovette tubes (Sarstedt, Nümbrecht, Germany) containing $0.106 \mathrm{~mol} / \mathrm{L}$ trisodium citrate in the absence and presence of corn trypsin inhibitor (CTI, Haematologic Technologies, Essex Junction, Vermont, United States; $1.45 \mu \mathrm{mol} / \mathrm{L}$, final concentration). The first collection tube was discarded. Whole blood rotational thromboelastometry (ROTEM) was performed directly after blood drawing. Platelet-rich plasma (PRP) was prepared by centrifugation at $150 \mathrm{~g}$ for 10 minutes at room temperature. Platelet count was adjusted to $150 \times 10^{9} / \mathrm{L}$ by dilution with autologous plateletpoor plasma (PPP). The latter was obtained by a second centrifugation step at $2,500 \mathrm{~g}$ for 15 minutes at room temperature. Storage of PPP in aliquots was at $-80^{\circ} \mathrm{C}$ until analysis. For assays performed with low TF levels, the CTI-containing blood was used, in order to exclude contribution of the contact activation pathway. ${ }^{22}$ The CTI concentration of $18.5 \mu \mathrm{g} / \mathrm{mL}(1.45 \mu \mathrm{mol} / \mathrm{L})$ was enough to inhibit FXIIa but avoid inhibition of FXIa. ${ }^{23}$

\section{Materials}

CTI was from Haematologic Technologies (Essex Junction, Vermont, United States); recombinant TF Innovin was from Dade Behring (Marburg, Germany); sulfatides and bovine serum albumin were from Sigma (St. Louis, Missouri, United States). Purified human FXII and FXI were obtained from Enzyme Research Laboratories (Stago, Leiden, the Netherlands). Recombinant tissue-type plasminogen activator(tPA) was from Boehringer Ingelheim (Alkmaar, the Netherlands). Synthetic phospholipids DOPS, DOPC, and DOPE (1,2-dioleoyl-sn-glycero3-phosphoserine, 1,2-dioleoyl-sn-glycero-3-choline, and 1,2-dioleoyl-sn-glycero-3-ethanolamine) were from Avanti Polar Lipids (Alabaster, Alabama, United States). Phospholipid vesicles (DOPS/DOPC/DOPE, 20/60/20 $\mathrm{mol} / \mathrm{mol} / \mathrm{mol}$ ) were prepared by sonication, as described earlier. ${ }^{24}$ Fluorogenic thrombin substrate, Z-Gly-Gly-Arg-aminomethyl coumarin (Z-GGR-AMC), was from Bachem (Bubendorf, Switzerland).

\section{Coagulation Assays}

Prothrombin time, aPTT, and levels of fibrinogen, antithrombin, and coagulation factors were measured on an ACL-TOP 750 system (Werfen, Le Pré-Saint-Gervais, France). The 
Table 1 General characteristics of the study population

\begin{tabular}{|c|c|c|c|}
\hline & Healthy individuals & FXI DEF patients & FXII DEF patients \\
\hline & $(n=10)$ & $(n=6)$ & $(n=4)$ \\
\hline Gender (female, \%) & $80 \%$ & $50 \%$ & $25 \%$ \\
\hline Age (years, mean $\pm S D$ ) & $48 \pm 10$ & $47 \pm 17$ & $34 \pm 16$ \\
\hline PT (s) [12-14] ${ }^{\mathrm{a}}$ & - & $13.0[13.0-15.6]$ & $13.0[13.0-13.3]$ \\
\hline aPTT (s) [28-35] & - & $66[46-282]^{\mathrm{b}}$ & $190[72-251]^{\mathrm{b}}$ \\
\hline FXI (IU/dL) & $96[86-124]$ & $3[<1 \text { to } 22]^{b, c}$ & 78 [73-125] \\
\hline FXII (IU/dL) & $101[60-141]$ & $100[93-108]$ & $2[<1 \text { to } 2]^{b, c}$ \\
\hline Prothrombin (IU/dL) & $95[85-110]$ & $102[89-124]$ & 98 [85-99] \\
\hline Fibrinogen $(\mathrm{g} / \mathrm{L})$ & $3.2[2.2-4.1]$ & $3.6[2.3-4.1]$ & $3.1[2.4-4.0]$ \\
\hline AT (IU/dL) & $113[102-134]$ & 110 [96-115] & 101 [96-112] \\
\hline FXIII activity (IU/dL) & $68[58-147]$ & 79 [56-85] & 87 [83-147] \\
\hline Plasminogen activity (IU/dL) & 101 [66-127] & 112 [100-135] & $97[86-131]$ \\
\hline tPA activity $(\mathrm{IU} / \mathrm{mL})$ & $0.5[0.5-0.7]$ & $0.5[0.4-0.5]$ & $0.6[0.5-0.7]$ \\
\hline PAl-1 activity (ng/mL) & $1.5[1.3-2.0]$ & $1.5[1.4-2.4]$ & $1.4[1.3-4.9]$ \\
\hline Alpha-2 antiplasmin (IU/dL) & $99[45-136]$ & $85[65-130]$ & $102[85-131]$ \\
\hline Platelets in PRP $\left(10^{9} / \mathrm{L}\right)$ & 309 [191-509] & $303[231-410]$ & 317 [197-429] \\
\hline
\end{tabular}

Abbreviations: aPTT, activated partial thromboplastin time; AT, antithrombin; DEF, deficient; FXI, factor XI; FXII, factor XII; PAI-1, plasminogen activator inhibitor-1; PRP, platelet-rich plasma; PT, prothrombin time; SD, standard deviation; tPA, tissue plasminogen activator.

Note: Data are presented as median and range [min-max] except for gender and age. $p$-Values were calculated for factor deficiency vs. control (Kruskall-Wallis ANOVA).

aeference values.

bIndicates that there is a significant difference between deficient patients and controls for the particular parameter $(p<0.05)$.

Individual FXI levels in FXI-deficient patients: $<1,<1,2,4,6,22 \mathrm{IU} / \mathrm{dL}$ and individual FXII levels in FXII-deficient patients: $<1,<1,2,2 \mathrm{IU} / \mathrm{dL}$.

standards, liquid antithrombin (HemosIL), QFA fibrinogen (HemosIL), recombiplastin prothrombin (HemosIL), and von Willebrand factor (VWF; AcuStar) were from Werfen. ELISA kits purchased from Hyphen Biomed (Nodia, Boom, Belgium) were used for activity tests of FXIII and PAI-1 (Zymutest), plasminogen and $\alpha 2$-antiplasmin (Biophen), and tPA (Zymuphen). All activity tests were performed in citrated plasma samples.

\section{Thrombin Generation Measurements}

The calibrated automated thrombogram (CAT) method (Diagnostica Stago, Asnieres sur Seine, France) was used to measure thrombin generation in PRP and PPP using 96-well plates. ${ }^{25}$ Briefly, per well $80 \mu \mathrm{L}$ plasma was added to $20 \mu \mathrm{L}$ of trigger consisting of sulfatides $(1 \mu \mathrm{mol} / \mathrm{L}$, f.c.) or $\mathrm{TF}(0.5 \mathrm{pmol} / \mathrm{L}$, f.c.). In the case of PPP, $4 \mu \mathrm{mol} / \mathrm{L}$ (f.c.) phospholipid vesicles were added to the trigger medium. Thrombin generation was started by adding $20 \mu \mathrm{L}$ of ZGGR-AMC (416 nmol/L) and $\mathrm{CaCl}_{2}(16.7 \mathrm{mmol} / \mathrm{L})$, and fluorescence accumulation was followed continuously in time. Platelet concentration in PRP was adjusted to $150 \times 10^{9} / \mathrm{L}$.

Where described, plasmas were supplemented with purified FXI ( 6 pmol in $200 \mu \mathrm{L}$ ) or FXII ( 75 pmol in $200 \mu \mathrm{L}$ ) on top of the residual level of FXI or FXII present in the plasma. To all plasma samples, the same amount of FXI or FXII, respectively, was added leading to an increase of the endogenous concentration with $100 \mathrm{IU} / \mathrm{dL}$.
From the thrombogram curves, the following parameters were obtained: lagtime ( $\mathrm{min}$ ), endogenous thrombin potential (ETP, $\left.\mathrm{nmol} / \mathrm{L} \mathrm{min}^{-1}\right)$, thrombin peak height $(\mathrm{nmol} / \mathrm{L})$, time-topeak (min), and the velocity index (VI, $\mathrm{nmol} / \mathrm{L} \mathrm{min}^{-1}$, equaling the thrombin peak height/[time to peak minus lagtime]).

\section{Whole Blood Thromboelastometry}

Thrombus elasticity parameters were determined by ROTEM (TEM International, Munich, Germany) to assess the build-up and lysis of a platelet-fibrin clot in whole blood. Measurements were performed at $37{ }^{\circ} \mathrm{C}$ using $300 \mu \mathrm{L}$ citrate-anticoagulated blood, which was triggered with $4 \mu \mathrm{mol} / \mathrm{L}$ sulfatides (f.c.) and $16 \mathrm{mmol} / \mathrm{L} \mathrm{CaCl}_{2}$ (intrinsic activation). Alternatively, the extrinsic pathway was triggered with $15 \mathrm{pmol} / \mathrm{L} \mathrm{TF}$ and $16 \mathrm{mmol} / \mathrm{L} \mathrm{CaCl}$ (CTI-containing blood). To measure clot lysis, tPA (125 ng/mL, f.c.) was added to the intrinsic or extrinsic reagent. ${ }^{26}$ Final concentrations of sulfatides, TF, and tPA were as optimized from prior dose-response curves. Where indicated, purified FXI or FXII was added to prewarmed blood samples, immediately before testing.

Where indicated, purified FXI ( $5.3 \mathrm{pmol}$ in $340 \mu \mathrm{L}$ ) or FXII (65 pmol in $340 \mu \mathrm{L}$ ) was added to prewarmed blood samples, immediately before testing. To all blood samples, the same amount of FXI or FXII was added, regardless of the residual level present in whole blood. Assuming a 50\% hematocrit, the supplemented amount of FXI or FXII resulted in an increase of the endogenous concentration with $100 \mathrm{IU} / \mathrm{dL}$. 
Evaluated parameters derived from thromboelasticity curves were: clotting time (CT), $\alpha$-angle (rate of clot formation), and maximal clot firmness (MCF). ${ }^{27}$ The clot lysis time (CLT 50\%) was calculated as the interval from 50\% clot formation to $50 \%$ clot lysis. Decay curve parameters further provided information on fibrinolysis at a later stage: the difference between lysis-onset time (LOT at $15 \%$ reduction of $\mathrm{MCF}$ ) and lysis time (LT at $90 \%$ reduction of MCF); fibrinolysis rate(FR) was defined as $75 \% /(\mathrm{LT}$ - LOT), which represents the curve decline in $\%$ per minute.

\section{Flow Experiments with Whole Blood}

Tendency of thrombus formation was determined by perfusion of recalcified citrated whole blood over a coated glass coverslip using the parallel-plate Maastricht chamber (dimensions flow channel: width $3 \mathrm{~mm}$, depth $50 \mu \mathrm{m}$, length $300 \mathrm{~mm}$ ). ${ }^{28}$ Glass coverslips were coated with microspots of collagen type I (applied at $50 \mu \mathrm{g} / \mathrm{mL}$ ) with or without $10 \mathrm{pg}$ applied recombinant TF, and used for flow perfusion assays, as described. ${ }^{29}$ Blood samples were prelabeled with $\mathrm{DiOC}_{6}(0.5 \mu \mathrm{g} / \mathrm{mL}$, f.c., stains mitochondria, vesicle membranes and endoplasmic reticulum of platelets and other cells), the phosphatidylserine probe Alexa Fluor (AF) 568-annexin A5 (1:200), and the fibrin precursor (AF)647fibrinogen $(16.5 \mu \mathrm{g} / \mathrm{mL}$, f.c.). The samples were co-infused with 10 vol\% coagulation medium (Hepes buffer pH 7.45 supplemented with $32 \mathrm{mmol} / \mathrm{L} \mathrm{MgCl}_{2}$ and $63 \mathrm{mmol} / \mathrm{L}$ $\mathrm{CaCl}_{2}$ ), using two pulse-free pumps. This resulted in physiological $(\mathrm{mmol} / \mathrm{L})$ concentrations of free $\mathrm{Ca}^{2+}$ and $\mathrm{Mg}^{2+}$. Wall-shear rate at the microspots was $1,000 \mathrm{~s}^{-1}$. Where indicated, purified FXI $(5.2 \mathrm{pmol}$ in $1000 \mu \mathrm{L})$ was added to blood samples, immediately before testing. To all blood samples, the same amount of FXI was added, regardless of the residual level present in whole blood. Assuming a 50\% hematocrit, the supplemented amount of FXI or FXII resulted in an increase of the endogenous concentration with $33 \mathrm{IU} / \mathrm{dL}$.

Brightfield microscopic images were captured at regular time intervals to monitor time-to-first-fibrin formation. After 11 minutes of blood perfusion, two-colored images were recorded using a fast line-scanning confocal Zeiss LSM7 system, equipped with a $63 \times$ oil-immersion objective (Carl Zeiss, Oberkochen, Germany). ${ }^{29}$ Confocal fluorescence images were analyzed by thresholding and assessment of surface area coverage above threshold, as before. ${ }^{30}$ At least five images were taken per microspot/color.

\section{Statistical Analysis}

Statistical analyses were performed with GraphPad Prism for Windows, version 6.0 (GraphPad Software, San Diego, California, United States). Paired data within patients were compared with the paired nonparametric Wilcoxon $t$-test. Parameters of thrombus formation between patients and controls were compared using a Kruskall-Wallis ANOVA with Dunn's post-test. Data are expressed as medians \pm interquartile ranges; data from individual patients are shown. $p$-Values $<0.05$ were considered to be statistically significant.

\section{Results}

Characteristics of the FXI- and FXII-Deficient Patients

In - Table 1 the general characteristics of the study population are shown. In all 10 adult patients, the PT was normal and the aPTT was prolonged, i.e., outside the normal range. FXI-deficient patients were normal in FXII levels, and vice versa. Plasma levels/activities of prothrombin, fibrinogen, antithrombin, and FXIII were within normal ranges and were comparable for patients and controls; the same applied to fibrinolysis parameters (plasminogen, tPA, PAI-1, and $\alpha 2$ antiplasmin). Since FXI deficiency (but not FXII deficiency) is sometimes associated with low levels of VWF, we determined the Von Willebrand ristocetin cofactor [vWF:RCo] activity in FXI-deficient individuals and healthy controls. The median vWF:RCo activity in FXI-deficient patients was $85 \mathrm{IU} / \mathrm{dL}$ (range: min-max, 65-130 IU/dL) and did not differ significantly from healthy controls (89 IU/dL [range: minmax, 49-164 IU/dL]). In addition, VWF activity and platelet counts were normal, thus ruling out quantitative VWFplatelet abnormalities.

\section{Assessing Impairments in Thrombin Generation Using PRP or PPP}

Thrombin-generation profiles were measured in the presence or absence of platelets. To specifically study contact activation we chose sulfatides, a component present in several tissues and compartments of the human body, which stimulates contact activation reactions more rapid than $\operatorname{kaolin}^{31}$ and generates coagulation activity. TF was used to trigger the extrinsic pathway. The optimal trigger concentrations were predetermined in normal plasmas (data not shown). When triggering plasmas from FXII-deficient patients with sulfatides, all thrombin generation parameters were prolonged (lagtime, time to thrombin peak) or reduced (peak height, ETP, VI), regardless of whether PRP or PPP was used (- Table 2). For the plasmas from FXI-deficient patients, only lagtime and time-to-thrombin peak were prolonged and were statistically significant in PRP (- Table 2).

In order to check that the changes in the thrombin generation parameters were a consequence of the factor deficiencies, purified FXI or FXII was added to the respective factor-deficient PRP samples, to reach a "normal" level; this process restored thrombin generation parameters upon triggering with sulfatides in PRP from all tested patients (-Fig. 1).

\section{Assessing Impairments in Platelet-Fibrin Elastic Clot Formation}

We assessed the formation and lysis of elastic clots consisting of aggregated, contracting platelets, and fibrin using ROTEM. ${ }^{26,32}$ - Table 3 shows the thromboelastogram parameters upon triggering with sulfatides and TF. Upon triggering with sulfatides, CTs were prolonged (CT, FXI-, and FXII-deficient patients) and curve slopes were reduced ( $\alpha$-angle, FXII-deficient patients) compared to controls. Thromboelastic curve parameters were not affected upon triggering with TF (- Table 3 ). Remarkably, for one of the FXII-deficient patients, thrombolysis parameters after sulfatide stimulation could not be calculated 
Table 2 Thrombin generation in platelet-rich plasma and platelet-poor plasma-contact pathway

\begin{tabular}{|c|c|c|c|}
\hline & Healthy controls & FXI DEF patients & FXII DEF patients $^{a}$ \\
\hline & $(n=10)$ & $(n=6)$ & $(n=4)$ \\
\hline \multicolumn{4}{|l|}{ Platelet-rich plasma } \\
\hline Lagtime (min) & $6.5[5.6-10.0]$ & $16.8[9.0-35.7]^{\mathrm{b}}$ & $55.1[17.0-120.0]^{\mathrm{b}}$ \\
\hline Time to peak (min) & $13.5[9.7-16.3]$ & $26.0[16.2-60.9]^{\mathrm{b}}$ & $77.2[30.5-120.0]^{\mathrm{b}}$ \\
\hline Peak height (nM) & $126[94-160]$ & $95[24-145]$ & $32[0-65]^{\mathrm{b}}$ \\
\hline ETP (nM min) & $1,502[1,252-1,612]$ & $1,525[894-2,069]$ & $280[0-1340]^{\mathrm{b}}$ \\
\hline $\mathrm{VI}(\mathrm{nM} / \mathrm{min})$ & $25.5[8.8-49.8]$ & $9.9[1.0-26.9]^{\mathrm{b}}$ & $1.5[0-4.9]^{\mathrm{b}}$ \\
\hline \multicolumn{4}{|l|}{ Platelet-poor plasma } \\
\hline Lagtime (min) & $6.0[3.7-9.7]$ & $11.3[3.7-29.3]$ & $36.2[12.8-51.3]^{\mathrm{b}}$ \\
\hline Time to peak (min) & $7.8[5.1-12.2]$ & $15.2[5.2-37.6]$ & $48.0[19.4-62.0]^{\mathrm{b}}$ \\
\hline Peak height (nM) & 293 [206-338] & 159 [6-398] & $8[6-47]^{\mathrm{b}}$ \\
\hline ETP (nM min) & $1,082[859-1,236]$ & $1,098[108-1,531]$ & $170[138-646]^{\mathrm{b}}$ \\
\hline $\mathrm{VI}(\mathrm{nM} / \mathrm{min})$ & $160.6[87.9-252.8]$ & $43.9[0.8-268.5]$ & $0.7[0.5-0.7]^{\mathrm{b}}$ \\
\hline
\end{tabular}

Abbreviations: DEF, deficient; ETP, endogenous thrombin potential; VI, velocity index.

Note: The platelet count was set to $150 \times 10^{9} / \mathrm{L}$ for platelet-rich plasma and $4 \mu \mathrm{M}$ phospholipids were added to the initiation mixtures for plateletpoor plasma. Data are presented as median and range [min-max]. p-Values were calculated for factor deficiency vs. control (Kruskall-Wallis ANOVA). ${ }^{a}$ In one FXII-deficient patient no measurable thrombin generation was observed (flat thrombin generation curve) in platelet-rich plasma. Therefore, the lagtime and time to peak were set to the maximum of 120 minutes. The peak height, ETP, and velocity index were set to a minimum of 0 . IIndicates that there is a significant difference between deficient patients and controls for the particular parameter $(p<0.05)$.

within 2 hours of measurement, because less than $90 \%$ of the thrombus was lysed within this time period. We also tested thrombolysis after stimulation of coagulation with TF and for this specific FXII-deficient patient the thrombolysis parameters were detectable but low at the selected concentration of $125 \mathrm{ng} / \mathrm{mL}$ tPA. Titration with higher amounts of tPA resulted in a normalized FR in this patient (-Supplementary Fig. S1).

To again confirm that any alterations in thromboelastometry curves were linked to the known factor deficiencies, residual patients' blood samples were supplemented with purified FXI or FXII. The added FXI or FXII normalized ROTEM parameters regardless of the trigger ( - Fig. 2). Of note, in the absence of tPA, no spontaneous clot lysis occurred within 2 hours for all tested blood samples (data not shown).

\section{Assessing Impairments in Whole Blood Formation of Fibrin-Thrombi under Flow}

To investigate the process of fibrin-thrombus formation under flow, blood samples from the FXI-deficient patients were preincubated with $\mathrm{DiOC}_{6}$ to label platelets, AF568annexin A5 to label phosphatidylserine-exposing platelets, and AF647-fibrinogen to stain formed fibrin fibers. Coperfusion of blood from patients with severe FXI deficiency with $\mathrm{CaCl}_{2} / \mathrm{MgCl}_{2}$ over microspots of collagen/TF for 11 minutes resulted in a substantial prolongation and suppression of fibrin formation, while this was not seen for blood from a patient with moderate FXI deficiency (FXI: 6 IU/dL) ( - Fig. 3). Staining for platelet deposition $\left(\mathrm{DiOC}_{6}\right)$ and phosphatidylserine exposure (AF568-annexin A5) at end-stage was in the same range for patients and control subjects. However, quantification of microscopic images indicated that, for the majority of patients, the time-to-fibrin formation was pro- longed, whilst the amount of fibrin formed at end-stage was reduced, in comparison to the controls (-Fig. 4A). These differences were most noticeable on collagen/TF microspots, but also apparent on collagen microspots, again suggesting that TF-induced triggering of the extrinsic system has a partly normalizing effect on a delayed intrinsic fibrinthrombus formation in these patients.

Remarkably, the blood samples from two patients with severely reduced FXI levels (1 and 4 IU/dL FXI) and a history of bleeding were most reduced and prolonged in fibrin formation, along with reduced platelet deposition. Increasing the endogenous FXI level in these two blood samples with $33 \mathrm{IU} / \mathrm{dL}$ improved the time-to-fibrin formation, platelet deposition and activation, along with fibrin formation on

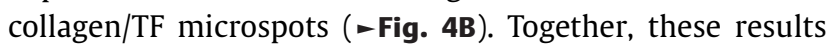
point to a diminished formation of platelet-fibrin thrombi under flow conditions for those patients with the most strongly reduced FXI level, and to a partial normalization of this process for FXI-deficient patients in the presence of TF.

\section{Integrative Comparison of Alterations in Thrombin Generation and Thromboelastometry}

To evaluate in more detail the changes in thrombin generation and thromboelastometry curve parameters, we produced colormaps to summarize the effects per patient (cf. residual FXI or FXII level, - Fig. 5); arbitrarily, normal ranges were set as means \pm 2 SD of the control group. These colormaps illustrate the powerful delay and reduction in sulfatide-triggered thrombin generation for both FXII- and FXI-deficient patients. Clots were formed later in the PRP and PPP samples for all FXIIdeficient patients in comparison to the plasmas from FXIdeficient patients, with only consistently strong delayed 


\section{PRP}
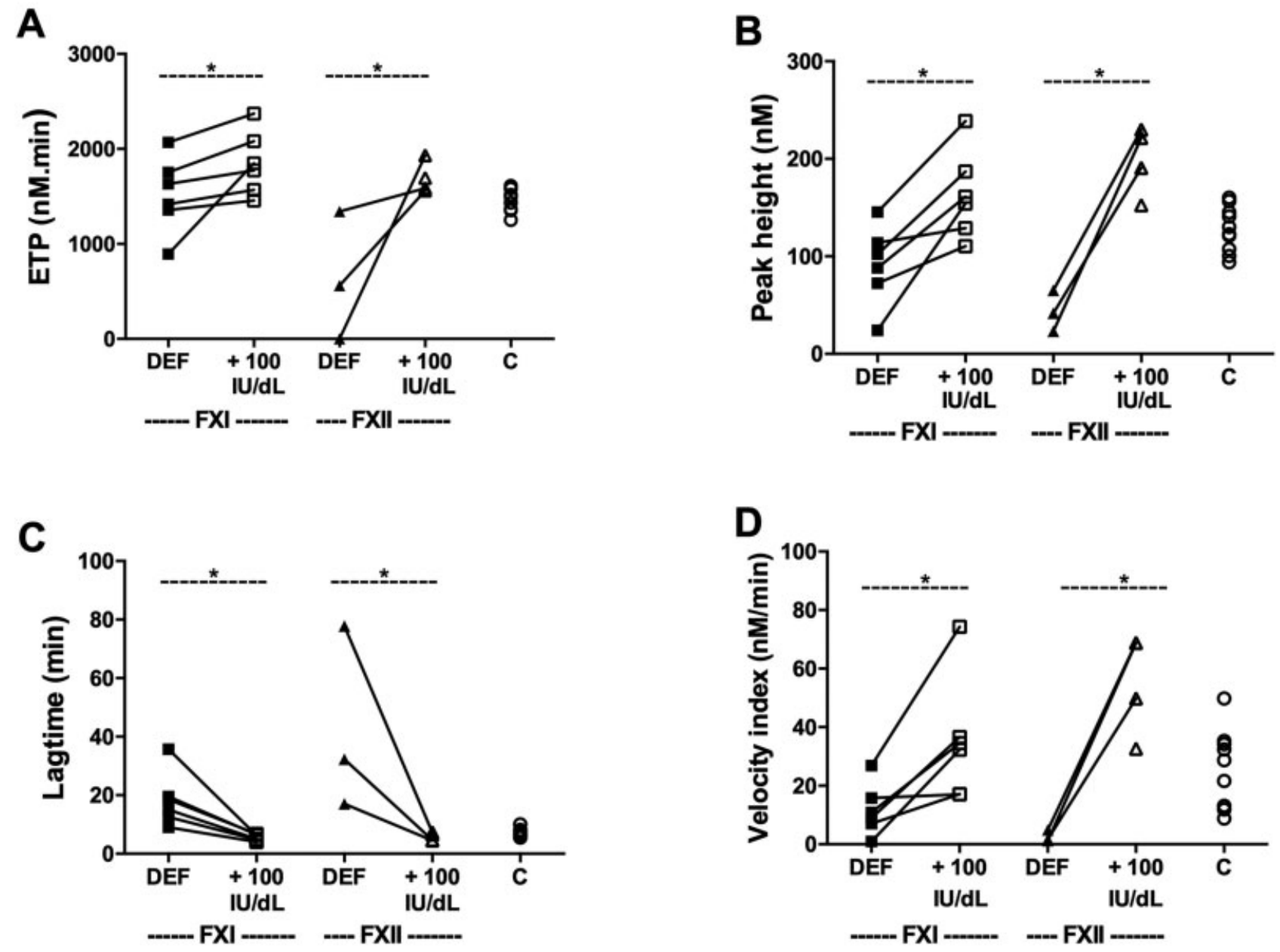

Fig. 1 Impaired thrombin generation after triggering the intrinsic pathway in platelet-rich plasma (PRP) from patients improved by factor addition. Thrombin generation in PRP from indicated controls (C) or FXI- or FXII-deficient patients. Triggering was with $\mathrm{CaCl}_{2}$ and $4 \mu \mathrm{mol} / \mathrm{L}$ sulfatides. Where indicated, purified FXI (6 pmol in $200 \mu \mathrm{L})$ or FXII (75 pmol in $200 \mu \mathrm{L})$ was preadded to PRP leading to an increase in the endogenous FXI or FXII concentration of $100 \mathrm{IU} / \mathrm{dL}$. In one FXII-deficient patient, no thrombin generation occurred in PRP without factor XII addition resulting in data $n=3$ in FXII DEF instead of $n=4$. The following curve parameters were assessed: ETP (endogenous thrombin potential) (A), thrombin peak height (B), lagtime to thrombin generation (C), and velocity index (D). ${ }^{*} p<0.05$ vs. no factor addition (Kruskal-Wallis ANOVA).

curves in PRP (- Fig. 5A, B). Interestingly, for all four FXIIdeficient patients, one or more markers of extent of thrombin generation (peak height, ETP, VI) were lower than in the control samples, whilst for most of the FXI-deficient patients these markers were not influenced, and in one out of six FXIdeficient patients these parameters were higher compared to controls.

Furthermore, the colormaps show the enhanced thrombin generation profiles in plasma samples for most patients upon reconstitution with purified FXI or FXII. Regarding thromboelastometry measurements, a consistent prolongation can be seen in kinetic parameters (FXII $>$ FXI), but only with sulfatides as a trigger. Furthermore, the tPA-dependent fibrinolysis was reduced in three out of six FXI-deficient patients ( - Fig. 5C), and was prolonged and reduced in one FXII-deficient patient (FXII $<1 \mathrm{IU} / \mathrm{dL}$ ), regardless of the trigger.

\section{Discussion}

Both in physiology (hemostasis) and pathophysiology (thrombosis), the coagulation cascade is thought to be triggered by TF. Under normal conditions minute amounts of TF, present on microvesicles, cell surfaces, or as part of the so-called "hemostatic envelope," drive a basal level of coagulation. In the present experiments we addressed the impact of FXII and FXI mainly by stimulating contact activation in blood or plasma obtained from patients with a congenital deficiency in either factor, versus controls.

First, in both thrombin generation and thromboelastometry measurements triggered with sulfatides, curve parameters were delayed and suppressed, as expected more in FXII than in FXI-deficient patients, when compared to the controls. Normalization of FXI or FXII factor levels in both PRP 
Table 3 ROTEM parameters-intrinsic and extrinsic pathway

\begin{tabular}{|c|c|c|c|c|c|c|}
\hline & \multicolumn{3}{|l|}{ Sulfatides } & \multicolumn{3}{|l|}{ TF } \\
\hline & $\begin{array}{l}\text { Healthy } \\
\text { controls }\end{array}$ & $\begin{array}{l}\text { FXI DEF } \\
\text { patients }\end{array}$ & $\begin{array}{l}\text { FXII DEF } \\
\text { patients }\end{array}$ & $\begin{array}{l}\text { Healthy } \\
\text { controls }\end{array}$ & $\begin{array}{l}\text { FXI DEF } \\
\text { patients }\end{array}$ & $\begin{array}{l}\text { FXII DEF } \\
\text { patients }\end{array}$ \\
\hline & $(n=10)$ & $(n=6)$ & $(n=4)$ & $(n=10)$ & $(n=6)$ & $(n=4)$ \\
\hline $\mathrm{CT}(\min )$ & $3.7[3.2-4.1]$ & $\begin{array}{l}5.1 \\
{[3.7-12.7]^{a}}\end{array}$ & $\begin{array}{l}27.9 \\
{[17.3-35.9]^{a}}\end{array}$ & $\begin{array}{l}4.8 \\
(2.8-6.7]\end{array}$ & $\begin{array}{l}3.3 \\
{[2.5-4.6]}\end{array}$ & $\begin{array}{l}5.5 \\
{[4.8-12.2]}\end{array}$ \\
\hline$\alpha$-angle (deg) & $\begin{array}{l}72.9 \\
{[64.5-79.0]}\end{array}$ & $\begin{array}{l}67.3 \\
{[31.0-75.0]}\end{array}$ & $\begin{array}{l}37.0 \\
{[18.0-49.0]^{\mathrm{a}}}\end{array}$ & $\begin{array}{l}69.5 \\
{[62.0-76.0]}\end{array}$ & $\begin{array}{l}72.5 \\
{[63.5-77.5]}\end{array}$ & $\begin{array}{l}68.5 \\
{[51.0-71.0]}\end{array}$ \\
\hline $\mathrm{MCF}(\mathrm{mm})$ & 55 [46-62] & 49 [32-55] & 24 [9-59] & $48[37-60]$ & 52 [41-55] & $44[26-64]$ \\
\hline $\begin{array}{l}\text { CLT 50\% } \\
(\min )\end{array}$ & $\begin{array}{l}26.5 \\
{[23.1-38.0]}\end{array}$ & $\begin{array}{l}33.3 \\
{[25.1-52.1]}\end{array}$ & $\begin{array}{l}24.8 \\
{[9.8-51.0]}\end{array}$ & $\begin{array}{l}26.5 \\
{[22.6-39.4]}\end{array}$ & $\begin{array}{l}31.0 \\
{[26.9-35.5]}\end{array}$ & $\begin{array}{l}25.8 \\
{[19.5-80.2]}\end{array}$ \\
\hline $\begin{array}{l}\text { FR } \\
\text { (\% decline/min) }\end{array}$ & $\begin{array}{l}5.3 \\
{[3.7-7.8]}\end{array}$ & $\begin{array}{l}3.3 \\
{[0.9-7.2]}\end{array}$ & $\begin{array}{l}6.2 \\
{[4.8-8.0]^{\mathrm{b}}}\end{array}$ & $\begin{array}{l}6.0 \\
{[2.0-9.2]}\end{array}$ & $\begin{array}{l}3.4 \\
{[2.5-6.7]}\end{array}$ & $\begin{array}{l}7.6 \\
{[1.4-9.4]}\end{array}$ \\
\hline
\end{tabular}

Abbreviations: CT, clotting time; DEF, deficient; MCF, maximum clot firmness; CLT 50\%, clot lysis time, interval from $50 \%$ clot formation to $50 \%$ clot lysis; FR, fibrinolysis rate; TF, tissue factor.

Note: Data are presented as median and range [min-max]. FR was calculated from the ROTEM parameters lysis-onset time (LOT) and lysis time (LT): $\mathrm{FR}=75 /(\mathrm{LT}-\mathrm{LOT})$ decline in \% per minute. $p$-Values: factor deficiency vs. control (Kruskall-Wallis ANOVA).

andicates that there is a significant difference between deficient patients and controls for the particular parameter $(p<0.05)$.

${ }^{b}$ In one FXII-deficient patient no LT and LOT could be calculated within 120 minutes and therefore no FR could be calculated.

and PPP in the intrinsic TG revealed normalization of the lagtime. Previously reported differences between FXI-deficient patients and controls were also determined from curves of TF-stimulated thrombin generation in PRP. Also, the prolonged lagtimes and reduced peaks in thrombin generation in bleeders versus nonbleeders and controls were observed upon triggering with TF most obvious in TG with CTI-treated PRP. ${ }^{33,34}$ In contrast, Zucker et al found no difference in TF-triggered TG measurements in FXI-deficient CTI-treated PPP. ${ }^{35}$

Remarkably, upon normalization of the factor level by adding purified FXI or FXII, some patients showed a higher thrombin generation (increased ETP and/or peak) than the controls ( - Fig. 5E, F). This increase was more pronounced in PRP than in PPP. This result indicates that the TG process (under stasis) can be enhanced beyond normal. In contrast, such enhancement was not seen in thromboelastometry experiments, suggesting that the rate of fibrin formation was already maximized under "normal" conditions. These findings confirm that additional thrombin is formed after clot formation. The amount of thrombin formed after fibrin formation exceeds the amount of thrombin needed for fibrin formation. ${ }^{36}$ These high TG data are in line with clinical experience reporting hypercoagulability and thrombotic complications in some patients with FXI deficiency when they receive replacement therapy with high-dose FXI. ${ }^{37}$ Moreover, epidemiological data show that high levels of FXI are a risk factor for venous thrombosis. ${ }^{38,39}$

In the presence of platelets, lagtime and time to peak in thrombin generation in FXI deficiency were more prolonged as compared to added phospholipids, which may indicate the importance of platelets in establishing a functional intrinsic system. Data from Kossmann et $\mathrm{al}^{18}$ showed that FXI activation occurs on platelets in a GPIb-V-IX-dependent manner, hence the degree of platelet activation may be a determinant in these reactions. From our data, the degree of platelet activation cannot be derived after triggering contact activation, although it can be assumed to play a role, given the formation of thrombin, a potent platelet activator.

Second, clot formation and the fibrinolytic aspects were tested in ROTEM experiments in the presence of tPA. Lower levels of FXII or FXI delayed clot formation in thromboelastometry upon triggering with sulfatides. In addition, the formed clots were smaller in FXII-deficient patients. Fibrinolysis did not differ between patient and controls; however, when we evaluated the patients individually, we observed that half of the FXIdeficient patients had a reduced FR. These same patients also had a decreased $\alpha$-angle indicating impairment in the build-up and breakdown of the clot. After the addition of purified FXI, the $\alpha$-angle was normal in all patients; however, more than half of FXI-deficient patients had a reduced FR with normal CLT 50\%. This indicates that the breakdown of the clot was impaired in the last part of fibrinolysis. In PRP and to a lesser extend in PPP, we observed an increased ETP in FXI-deficient patients after reconstitution with purified FXI. The formation of thrombin inside the clot protects against fibrinolysis possibly explaining this reduction in FR. ${ }^{36}$ These data support a contributory role of FXII and FXI in clot formation upon contact activation, as well as a role in clot lysis. Thromboelastography has been used to investigate coagulation in FXI-deficient patients. However, these publications used the commercial INTEM reagent which uses ellagic acid (which is not a physiological trigger) to activate FXII or a low concentration of TF; fibrinolysis was often not investigated and plasma instead of whole blood has been used. ${ }^{40-42}$

The net effect of FXI and FXII on the balance between clot formation and breakdown is complex. Earlier studies in humans show an effect of FXII on fibrinolysis, with subjects deficient in FXII having a diminished clot lysis. ${ }^{43}$ Furthermore, activation of coagulation by FXII/FXI drives thrombin formation 


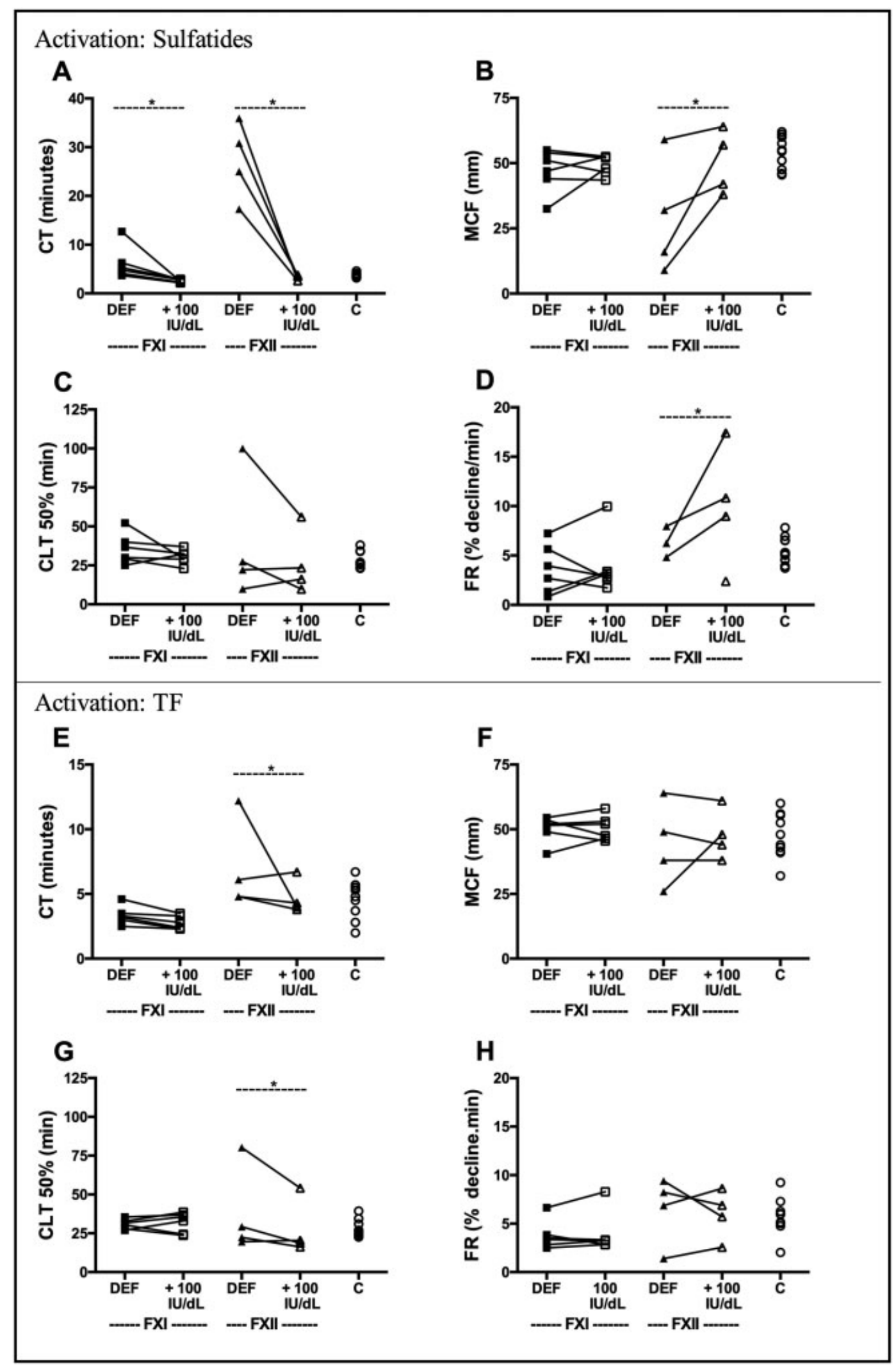

Fig. 2 Aberrant elastic clot formation in whole blood from patients normalized by factor addition. ROTEM curves were generated with whole blood from indicated controls (C) or FXI- or FXII-deficient patients. Triggering was with $\mathrm{CaCl}_{2}$ and $4 \mu \mathrm{mol} / \mathrm{L}$ sulfatides $\left(\mathrm{A}-\mathrm{D}\right.$ ), or $\mathrm{CaCl}_{2}$ and $15 \mathrm{pmol} / \mathrm{LTF}$ (CTI-treated blood) $(\mathrm{E}-\mathrm{H})$. Where indicated, $\mathrm{FXI}(5.3 \mathrm{pmol}$ in $340 \mu \mathrm{L})$ or FXII $(65 \mathrm{pmol}$ in $340 \mu \mathrm{L})$ was preadded to the blood leading to an increase in the endogenous FXI or FXII concentration of $100 \mathrm{IU} / \mathrm{dL}$. For one FXII-deficient patient, the fibrinolysis rate after sulfatide stimulation could not be calculated (D) since lysis velocity was extremely slow and less than $90 \%$ of the thrombus was lysed within 2 hours of measurement. The following curve parameters were assessed: $C T$ (clotting time), MCF (maximal clot firmness), CLT ( $50 \%$ clot lysis time), FR (fibrinolysis rate). ${ }^{*} p<0.05$ vs. no factor addition (Kruskal-Wallis ANOVA). CTI, corn trypsin inhibitor; TF, tissue factor. 


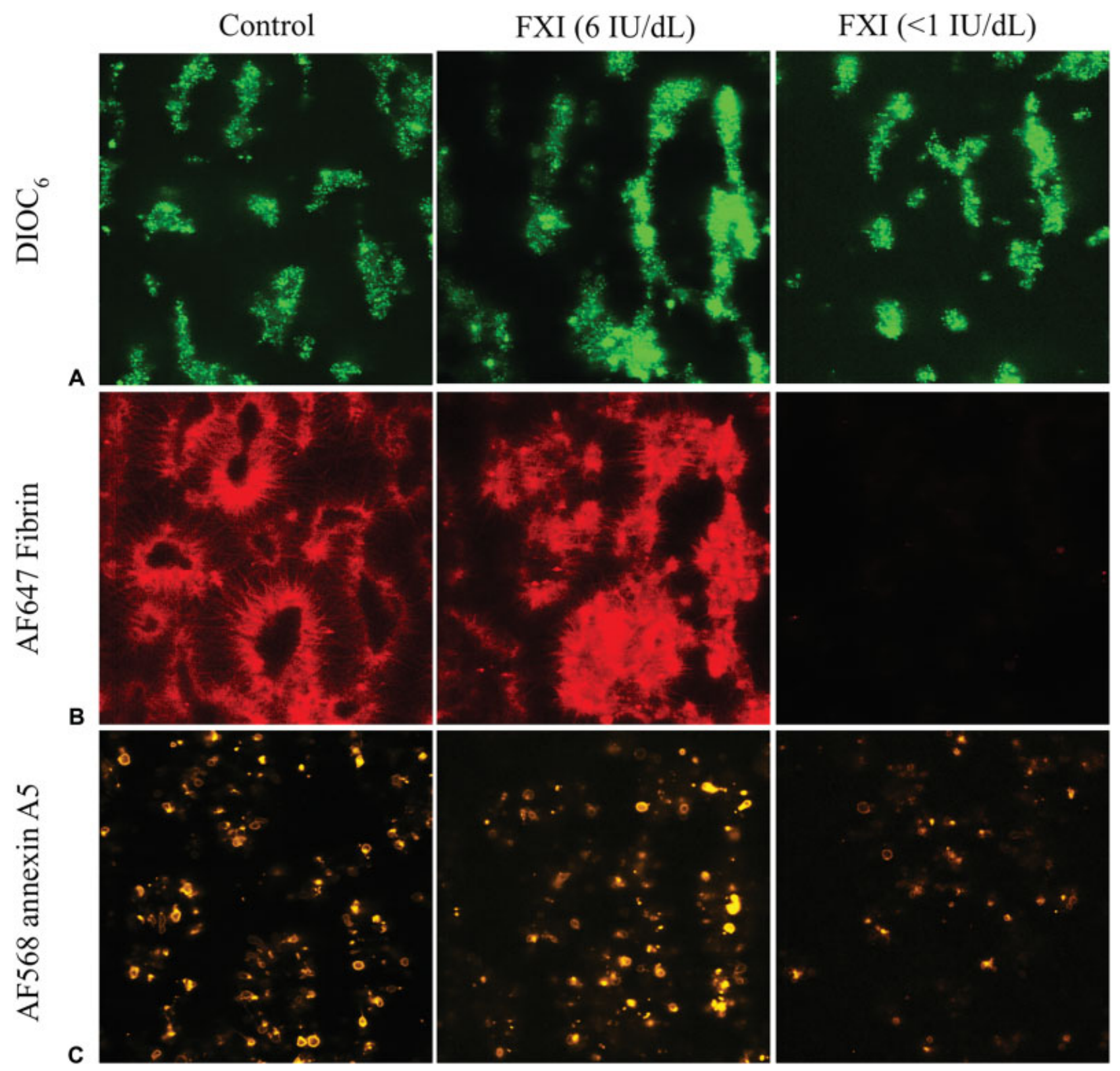

Fig. 3 Impaired fibrin-thrombus formation in whole blood from patients with factor XI deficiency. Whole blood samples from representative controls and indicated patients (FXI levels $6 \mathrm{IU} / \mathrm{dL}$ and $<1 \mathrm{IU} / \mathrm{dL}$, respectively) were perfused under recalcification over microspots of collagen $\pm T F$ at a wall-shear rate of $1,000 \mathrm{~s}^{-1}$ for 11 minutes. Real-time recorded brightfield microscopic images were analyzed for fibrinthrombus formation (-Fig. 4). Fluorescence images at endstage were taken for measurement of platelet deposition (A, DiOC 6 ), AF647-fibrin formation (B), and phosphatidylserine exposure (C, AF568-annexin A5). Representative images $(106 \times 106 \mu \mathrm{m})$ are shown, captured from microspots of collagen/TF. TF, tissue factor.

which in turn is an important determinant of clot strength and fibrinolysis, among others by activation of FXIII and TAFI. ${ }^{44}$ Part of this complexity may also explain the nonlinear effects of FXII activity in relation to arterial thrombotic events.

Replenishing of factors shortened clot formation upon FXI correction and CLT (CLT 50\%) measured to 50\% degradation of the clot was normalized.

Upon TF activation (in CTI-containing blood), the thromboelastometry parameters showed less remarkable differences between FXII- or FXI-deficient patients and controls. These results are in line with a previous study where upon TF triggering using thromboelastometry no significant difference in parameters was seen between FXI-deficient patients when measured in CTI-treated whole blood. ${ }^{41}$ Only in one FXIIdeficient patient (FXII $<1 \mathrm{IU} / \mathrm{dL}$ ) fibrinolysis was delayed and decreased in thromboelastometry, although more pronounced after sulfatide triggering. In this patient sample, higher tPA levels were needed to lyse the clot formed. Here, it is relevant to note that in a case report another severe FXII-deficient patient with diminished fibrinolysis was reported who experienced a myocardial infarction. ${ }^{45}$

Third, under flow conditions using whole blood samples, triggered with collagen \pm TF to stimulate fibrin-thrombus formation, in the majority of FXI-deficient patients the time-to- 


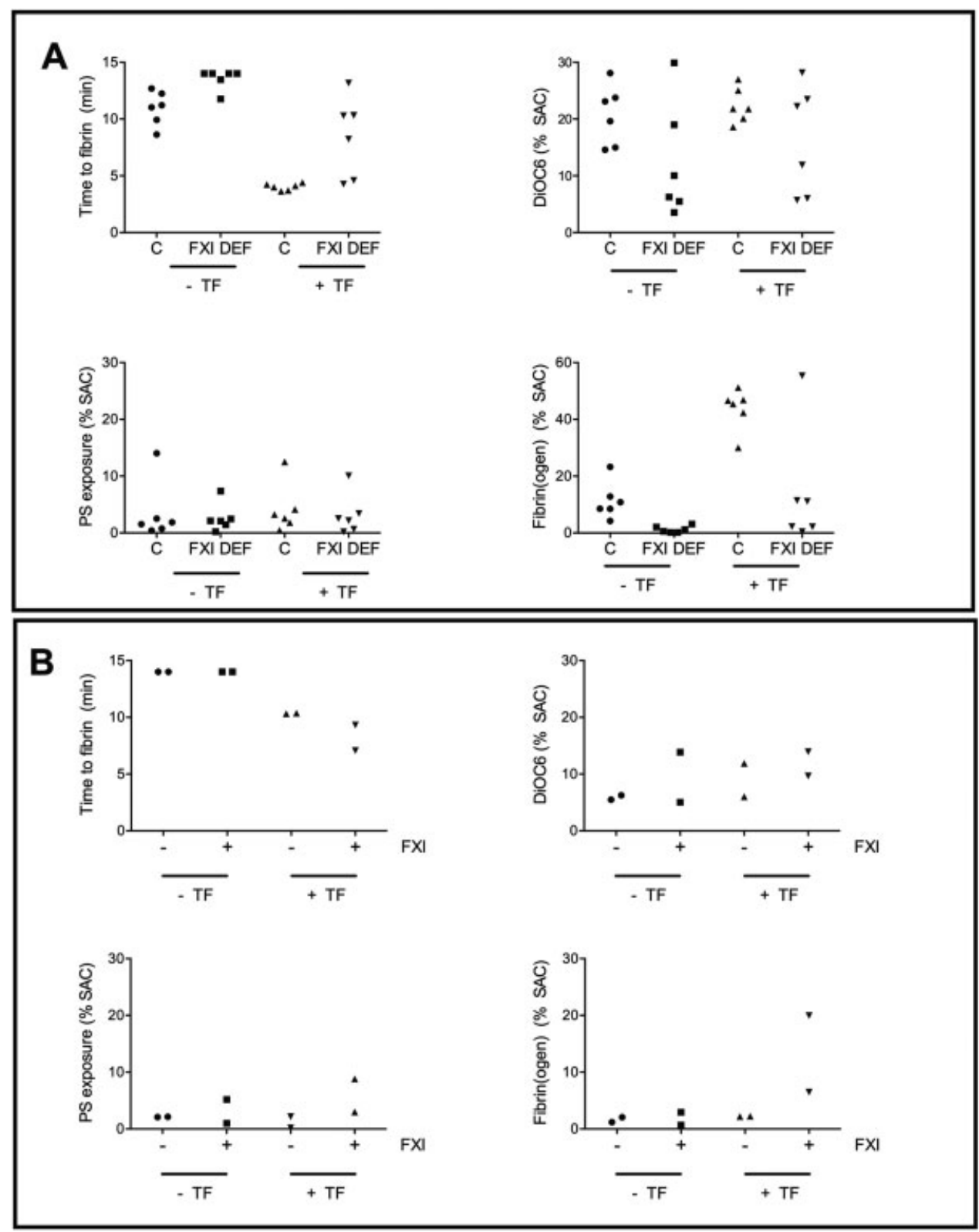

Fig. 4 Quantification of fibrin-thrombus formation in whole blood from patients with factor XI deficiency. Whole blood samples from control subjects and indicated FXI-deficient patients were perfused under recalcification over microspots of collagen $\pm T F$ for 11 minutes, as described for $\boldsymbol{\sim}$ Fig. 3. Microscopic images were analyzed for: time-to-first fibrin formation, platelet deposition (DiOC 6 , \%SAC), phosphatidylserine exposure (AF568-annexin A5, \%SAC), and AF647-fibrin formation (\%SAC) (A). Furthermore, blood samples from two FXI-deficient patients ( 4 or $<1 \mathrm{IU} / \mathrm{dL}$ ) were supplemented with purified FXI $(5.2 \mathrm{pmol}$ in $1,000 \mu \mathrm{L}$ ) leading to an increase in the endogenous FXI concentration of $33 \mathrm{lU} / \mathrm{dL}$ and used for the same perfusion assay. Microscopic images were again analyzed for: time-to-first fibrin formation, platelet deposition ( $\mathrm{BiOC}_{6}$ ), phosphatidylserine (AF568-annexin A5), and AF647-fibrin formation (B). TF, tissue factor.

fibrin formation was delayed and fibrin formed was reduced; however, there was hardly a difference in platelet deposition and phosphatidylserine exposure. Previous work has shown a key role of immobilized collagen in enhancing coagulation via the FXI/FXII pathway. ${ }^{46,47}$ Zhu et al also found in flow experiments where FXI antibodies 14E11 or 01A6 were added to blood of healthy individuals efficiently abolished thrombin and fibrin generation without affecting platelet deposition on the collagen surface. ${ }^{48}$ They did not measure phosphatidylserine exposure. Our result was confirmed with confocal fluorescence images and interestingly also indicates that the degree of FXI deficiency is important, since a severe
FXI-deficient patient ( $<1 \mathrm{IU} / \mathrm{dL}$ ) showed an impaired thrombus and fibrin formation when compared with a moderate FXI-deficient patient $(6 \mathrm{IU} / \mathrm{dL})$ and a control. Addition of purified FXI in the blood samples of two FXI-deficient patients marked as severe bleeders improved thrombus formation and activation of platelets in a flow experiment on collagen/TF microspots. Although we could only assess one severe FXII-deficient patient in these flow experiments, both in the absence or presence of TF the platelet deposition was diminished (data not shown). The available data indicate an important role for the thrombin-FXI feedback loop involving platelets, in TF/collagen-dependent thrombus formation under 


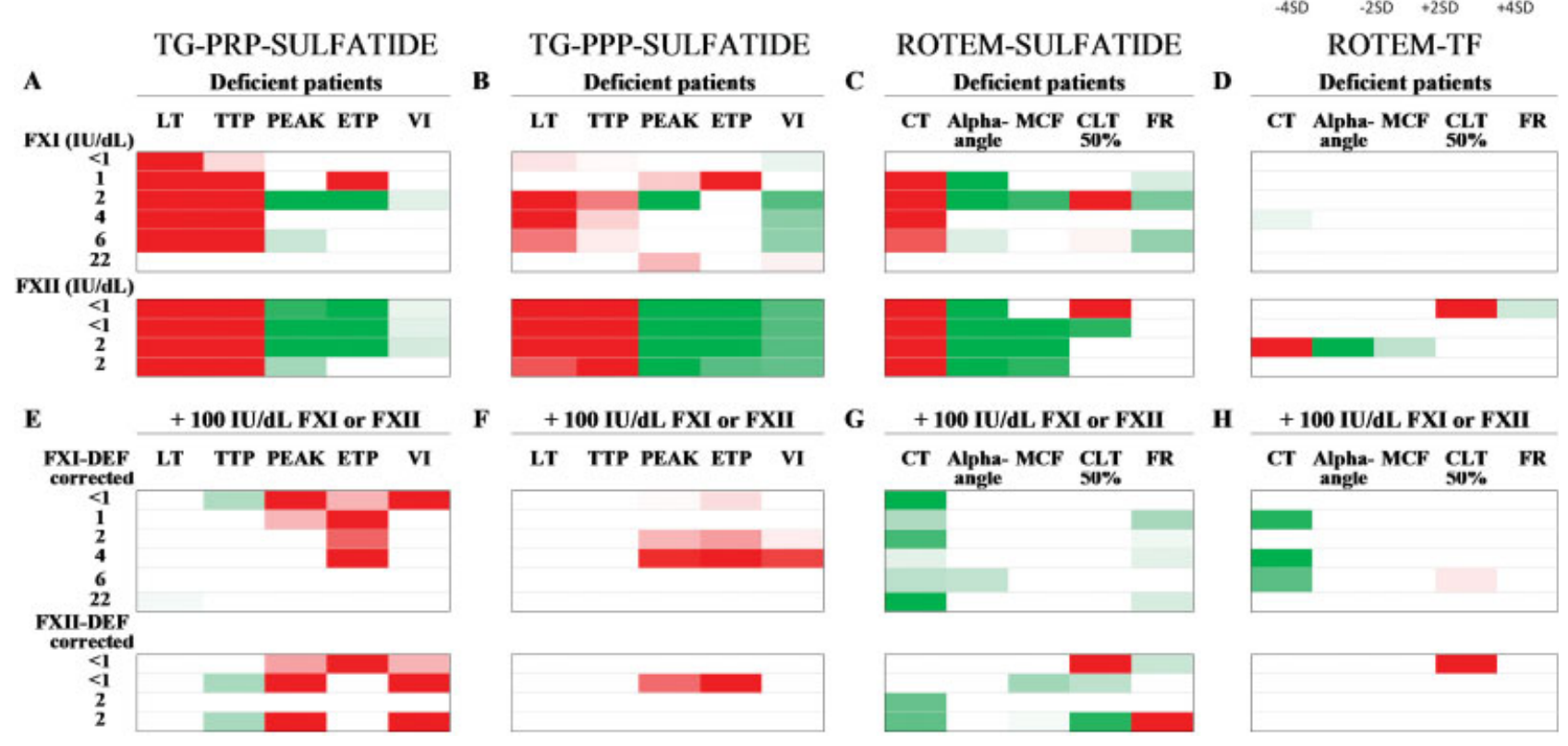

Fig. 5 Comparative analysis of alterations in thrombin generation and thromboelastometry for individual patients. Colormaps of relevant increased (red) or decreased (green) values of indicated curve parameters of thrombin generation in PRP ( $A$ and $E$ ), thrombin generation in PPP (B and F), and thromboelastometry ( $C-D$ and $G-H)$. Altered values were considered to be relevant, if outside the range of mean \pm 2 SD of values from the control group (white). Borders for conditional formatting are mean \pm 2 SD and mean \pm 4 SD. Individual patients were ordered per line, ranking from higher to lower deficiency (factor levels indicated). Basal values (upper panels) and effects of factor repletion (lower panels) after triggering with sulfatides or TF as indicated in the figure (C). $\alpha$-angle, rate of clot formation; CLT $50 \%$, clot lysis time calculated as the interval from $50 \%$ clot formation to $50 \%$ clot lysis (s); CT, clotting time (s); ETP, endogenous thrombin potential (nM min); FR, fibrinolysis rate between lysisonset time (LOT at $15 \%$ reduction of MCF) and lysis time (LT at $90 \%$ reduction of MCF) (\% decline/min); LT, lagtime (min); MCF, maximal clot firmness (mm); PEAK (mm); PPP, platelet-poor plasma; PRP, platelet-rich plasma; SD, standard deviation; TTP, time to peak (s); VI, velocity index $(\mathrm{nM} / \mathrm{min})$.

flow. This also strengthens the notion that FXI is involved in the initial hemostatic clot forming process, which helps to explain the mild bleeding disorder in FXI-deficient subjects. As established before, deficiency in FVIII or FIX (associated with major bleeding episodes) caused a much larger suppressing effect on the fibrin formation under flow. ${ }^{49}$

The main limitations of this study concern the low number of subjects available in one center, also due to the low prevalence of these deficiencies. Another limitation is that the degree of variation in test outcomes does not allow for definitive conclusions on associations between coagulation responses on the one hand and bleeding tendency on the other hand.

In conclusion, in thrombin generation tested in sulfatideactivated plasma, or sulfatide-activated blood tested in ROTEM, deficiency of FXI or FXII had a substantial effect on thrombin and clot formation respectively, including a remarkable and late occurring delay in clot lysis, in most FXI-deficient patients. Furthermore, clot formation induced by TF/collagen was strongly determined by the residual level of FXI in the patient's blood and was corrected by the addition of purified FXI. The residual FXI level does not always determine the bleeding severity. Therefore, the impact of a congenital FXI deficiency on bleeding tendencies and treatment of individuals with thrombotic disorders with inhibitors of FXIa or FXIIa should be further investigated to better understand the impact on hemostasis and the potential bleeding risk associated with such interventions.

\section{Authors' Contributions}

H.t.C., Y.D., J.W.P.G.-R., and J.K. were responsible for overall study conception and design; J.W.P.G-R. supervised the research, assisted in experiments, interpreted data, performed statistical analysis, and wrote the manuscript; J.K. and J.P.v.G. performed experiments and analyzed the data; J.M.E.M.C. designed flow experiments, performed statistical analysis, and interpreted data; B.d.L. was involved in initiation of the study and edited the manuscript; J.W.M.H. and H.t.C. contributed to manuscript writing, critically reviewed the manuscript, and interpreted data; Y.D. enrolled the patients, collected clinical data, and performed initial analysis of patient and sample characteristics. All authors approved the final version of the manuscript.

\section{Funding}

This study was supported by the Cardiovascular Center (HVC) of Maastricht University Medical Centre, the Hemker foundation (CARIM), CSL Behring France, ADRHEC, the Dutch Heart Foundation (2015T79 to J.M.E.M.C.), the Netherlands, and the Organization for Scientific Research (NWO Vidi 91716421 to J.M.E.M.C). 


\section{Conflict of Interest}

J.K. and B.d.L. are employees of Synapse Research Institute, Maastricht, the Netherlands, but declare to have no conflict of interests. J.H. is co-founder and shareholder of FlowChamber. All other authors declare no competing financial interests. Dr. ten Cate reports personal fees from Advisory Board Bayer, grants from Contract research: Bayer, Pfizer/BMS, during the conduct of the study.

\section{Acknowledgements}

We thank Dr. Patty Nelemans for statistical support and Dr. Frauke Swieringa for analysis of flow experiments.

\section{References}

1 Miller GJ, Esnouf MP, Burgess AI, Cooper JA, Mitchell JP. Risk of coronary heart disease and activation of factor XII in middleaged men. Arterioscler Thromb Vasc Biol 1997;17(10): 2103-2106

2 Doggen CJ, Rosendaal FR, Meijers JC. Levels of intrinsic coagulation factors and the risk of myocardial infarction among men: opposite and synergistic effects of factors XI and XII. Blood 2006; 108(13):4045-4051

3 Govers-Riemslag JW, Smid M, Cooper JA, et al. The plasma kallikrein-kinin system and risk of cardiovascular disease in men. J Thromb Haemost 2007;5(09):1896-1903

4 Siegerink B, Govers-Riemslag JWP, Rosendaal FR, Ten Cate H, Algra A. Intrinsic coagulation activation and the risk of arterial thrombosis in young women: results from the Risk of Arterial Thrombosis in relation to Oral contraceptives (RATIO) case-control study. Circulation 2010;122(18):1854-1861

5 Renné T, Pozgajová $M$, Grüner $S$, et al. Defective thrombus formation in mice lacking coagulation factor XII. JExp Med 2005;202(02):271-281

6 Kleinschnitz C, Stoll G, Bendszus M, et al. Targeting coagulation factor XII provides protection from pathological thrombosis in cerebral ischemia without interfering with hemostasis. J Exp Med 2006;203(03):513-518

7 Lorentz CU, Verbout NG, Cao Z, et al. Factor XI contributes to myocardial ischemia-reperfusion injury in mice. Blood Adv 2018; 2(02):85-88

8 Zhang $\mathrm{H}$, Löwenberg EC, Crosby JR, et al. Inhibition of the intrinsic coagulation pathway factor XI by antisense oligonucleotides: a novel antithrombotic strategy with lowered bleeding risk. Blood 2010;116(22):4684-4692

9 Revenko AS, Gao D, Crosby JR, et al. Selective depletion of plasma prekallikrein or coagulation factor XII inhibits thrombosis in mice without increased risk of bleeding. Blood 2011;118(19): 5302-5311

10 Matafonov A, Leung PY, Gailani AE, et al. Factor XII inhibition reduces thrombus formation in a primate thrombosis model Blood 2014;123(11):1739-1746

11 van Montfoort ML, Knaup VL, Marquart JA, et al. Two novel inhibitory anti-human factor XI antibodies prevent cessation of blood flow in a murine venous thrombosis model. Thromb Haemost 2013;110(05):1065-1073

12 Crosby JR, Marzec U, Revenko AS, et al. Antithrombotic effect of antisense factor XI oligonucleotide treatment in primates. Arterioscler Thromb Vasc Biol 2013;33(07):1670-1678

13 Krupka J, May F, Weimer T, et al. The coagulation factor XIIa inhibitor rHA-Infestin-4 improves outcome after cerebral ischemia/reperfusion injury in rats. PLoS One 2016;11(01):e0146783

14 Girolami A, Candeo N, De Marinis GB, Bonamigo E, Girolami B. Comparative incidence of thrombosis in reported cases of deficiencies of factors of the contact phase of blood coagulation. J Thromb Thrombolysis 2011;31(01):57-63
15 Büller HR, Bethune C, Bhanot S, et al; FXI-ASO TKA Investigators. Factor XI antisense oligonucleotide for prevention of venous thrombosis. N Engl J Med 2015;372(03):232-240

16 Versteeg HH, Heemskerk JW, Levi M, Reitsma PH. New fundamentals in hemostasis. Physiol Rev 2013;93(01):327-358

17 Kravtsov DV, Matafonov A, Tucker EI, et al. Factor XI contributes to thrombin generation in the absence of factor XII. Blood 2009;114 (02):452-458

18 Kossmann S, Lagrange J, Jäckel S, et al. Platelet-localized FXI promotes a vascular coagulation-inflammatory circuit in arterial hypertension. Sci Transl Med 2017;9(375):eaah4923

19 Colman RW, Schmaier AH. Contact system: a vascular biology modulator with anticoagulant, profibrinolytic, antiadhesive, and proinflammatory attributes. Blood 1997;90(10):3819-3843

20 Konings J, Govers-Riemslag JW, Philippou H, et al. Factor XIIa regulates the structure of the fibrin clot independently of thrombin generation through direct interaction with fibrin. Blood 2011;118 (14):3942-3951

21 Konings J, Hoving LR, Ariëns RS, et al. The role of activated coagulation factor XII in overall clot stability and fibrinolysis. Thromb Res 2015;136(02):474-480

22 Dargaud Y, Luddington R, Baglin TP. Elimination of contact factor activation improves measurement of platelet-dependent thrombin generation by calibrated automated thrombography at lowconcentration tissue factor. JThromb Haemost 2006;4(05): 1160-1161

23 Hansson KM, Nielsen S, Elg M, Deinum J. The effect of corn trypsin inhibitor and inhibiting antibodies for FXIa and FXIIa on coagulation of plasma and whole blood. J Thromb Haemost 2014;12(10): 1678-1686

24 Govers-Riemslag JWP, Janssen MP, Zwaal RF, Rosing J. Effect of membrane fluidity and fatty acid composition on the prothrombin-converting activity of phospholipid vesicles. Biochemistry 1992;31(41):10000-10008

25 Hemker HC, Giesen P, Al Dieri R, et al. Calibrated automated thrombin generation measurement in clotting plasma. Pathophysiol Haemost Thromb 2003;33(01):4-15

26 Kuiper GJ, Kleinegris MC, van Oerle R, et al. Validation of a modified thromboelastometry approach to detect changes in fibrinolytic activity. Thromb J 2016;14:1-13

27 MacDonald SG, Luddington RJ. Critical factors contributing to the thromboelastography trace. Semin Thromb Hemost 2010;36(07): 712-722

28 Van Kruchten R, Cosemans JM, Heemskerk JW. Measurement of whole blood thrombus formation using parallel-plate flow chambers: a practical guide. Platelets 2012;23(03):229-242

29 Swieringa F, Baaten CC, Verdoold R, et al. Platelet control of fibrin distribution and microelasticity in thrombus formation under flow. Arterioscler Thromb Vasc Biol 2016;36(04):692699

30 de Witt SM, Swieringa F, Cavill R, et al. Identification of platelet function defects by multi-parameter assessment of thrombus formation. Nat Commun 2014;5:4257-4269

31 Tans G, Griffin JH. Properties of sulfatides in factor-XII-dependent contact activation. Blood 1982;59(01):69-75

32 de Witt SM, Verdoold R, Cosemans JM, Heemskerk JW. Insights into platelet-based control of coagulation. Thromb Res 2014;133 (Suppl 2):S139-S148

33 Rugeri L, Quélin F, Chatard B, De Mazancourt P, Negrier C, Dargaud Y. Thrombin generation in patients with factor XI deficiency and clinical bleeding risk. Haemophilia 2010;16 (05):771-777

34 Pike GN, Cumming AM, Hay CR, Bolton-Maggs PH, Burthem J. Sample conditions determine the ability of thrombin generation parameters to identify bleeding phenotype in FXI deficiency. Blood 2015;126(03):397-405

35 Zucker M, Seligsohn U, Salomon O, Wolberg AS. Abnormal plasma clot structure and stability distinguish bleeding risk in patients 
with severe factor XI deficiency. JThromb Haemost 2014;12(07): $1121-1130$

36 von dem Borne PA, Meijers JC, Bouma BN. Feedback activation of factor XI by thrombin in plasma results in additional formation of thrombin that protects fibrin clots from fibrinolysis. Blood 1995; 86(08):3035-3042

37 Batty P, Honke A, Bowles L, et al. Ongoing risk of thrombosis with factor XI concentrate: 5 years experience in two centres. Haemophilia 2015;21(04):490-495

38 Meijers JC, Tekelenburg WL, Bouma BN, Bertina RM, Rosendaal FR. High levels of coagulation factor XI as a risk factor for venous thrombosis. N Engl J Med 2000;342(10):696-701

39 Cushman M, O'Meara ES, Folsom AR, Heckbert SR. Coagulation factors IX through XIII and the risk of future venous thrombosis: the Longitudinal Investigation of Thromboembolism Etiology. Blood 2009;114(14):2878-2883

40 Livnat T, Shenkman B, Martinowitz U, et al. The impact of thrombin generation and rotation thromboelastometry on assessment of severity of factor XI deficiency. Thromb Res 2015;136(02):465-473

41 Pike GN, Cumming AM, Thachil J, Hay CRM, Bolton-Maggs PHB, Burthem J. Evaluation of the use of rotational thromboelastometry in the assessment of FXI deficency. Haemophilia 2017;23 (03):449-457

42 Kitchen DP, Kitchen S, Jennings I, Woods T, Walker I. Quality assurance and quality control of thrombelastography and rotational thromboelastometry: the UK NEQAS for blood coagulation experience. Semin Thromb Hemost 2010;36(07):757-763
43 Levi M, Hack CE, de Boer JP, Brandjes DP, Büller HR, ten Cate JW. Reduction of contact activation related fibrinolytic activity in factor XII deficient patients. Further evidence for the role of the contact system in fibrinolysis in vivo. JClin Invest 1991;88(04): $1155-1160$

44 Mosnier LO, Bouma BN. Regulation of fibrinolysis by thrombin activatable fibrinolysis inhibitor, an unstable carboxypeptidase B that unites the pathways of coagulation and fibrinolysis. Arterioscler Thromb Vasc Biol 2006;26(11):2445-2453

45 Lodi S, Isa L, Pollini E, Bravo AF, Scalvini A. Defective intrinsic fibrinolytic activity in a patient with severe factor XII-deficiency and myocardial infarction. Scand J Haematol 1984;33(01):80-82

46 van der Meijden PE, Munnix IC, Auger JM, et al. Dual role of collagen in factor XII-dependent thrombus formation. Blood 2009;114(04):881-890

47 Kuijpers MJ, van der Meijden PE, Feijge MA, et al. Factor XII regulates the pathological process of thrombus formation on ruptured plaques. Arterioscler Thromb Vasc Biol 2014;34(08):1674-1680

48 Zhu S, Travers RJ, Morrissey JH, Diamond SL. FXIa and platelet polyphosphate as therapeutic targets during human blood clotting on collagen/tissue factor surfaces under flow. Blood 2015; 126(12):1494-1502

49 Swieringa F, Kuijpers MJ, Lamers MM, van der Meijden PE, Heemskerk JW. Rate-limiting roles of the tenase complex of factors VIII and IX in platelet procoagulant activity and formation of platelet-fibrin thrombi under flow. Haematologica 2015;100 (06):748-756 